\title{
Structure and Dynamics of Glycosphingolipids in Lipid Bilayers: Insights from Molecular Dynamics Simulations
}

\author{
Ronak Y. Patel ${ }^{1,2}$ and Petety V. Balaji ${ }^{1}$ \\ ${ }^{1}$ Department of Biosciences and Bioengineering, Indian Institute of Technology Bombay, Powai, Mumbai 400 076, India \\ ${ }^{2}$ Department of Genetics, Washington University School of Medicine, St. Louis, MO 63110, USA
}

Correspondence should be addressed to Petety V. Balaji, balaji@iitb.ac.in

Received 7 September 2010; Revised 3 January 2011; Accepted 14 February 2011

Academic Editor: Richard D. Cummings

Copyright ( $) 2011$ R. Y. Patel and P. V. Balaji. This is an open access article distributed under the Creative Commons Attribution License, which permits unrestricted use, distribution, and reproduction in any medium, provided the original work is properly cited.

\begin{abstract}
Glycolipids are important constituents of biological membranes, and understanding their structure and dynamics in lipid bilayers provides insights into their physiological and pathological roles. Experimental techniques have provided details into their behavior at model and biological membranes; however, computer simulations are needed to gain atomic level insights. This paper summarizes the insights obtained from MD simulations into the conformational and orientational dynamics of glycosphingolipids and their exposure, hydration, and hydrogen-bonding interactions in membrane environment. The organization of glycosphingolipids in raft-like membranes and their modulation of lipid membrane structure are also reviewed.
\end{abstract}

\section{Glycolipids}

Glycans are covalently attached to either a glycerol or a sphingosine backbone forming a glycophospho (glycoglycero) or glycosphingolipid, respectively $[1,2]$. These glycolipids are amphipathic molecules that are anchored in lipid bilayers through their lipid moiety. They are ubiquitous components of plasma membranes of all vertebrate cells and, recently, have been found to be present on nuclear envelope as well [3]. Although glycosphingolipids are distributed in a wide variety of tissues, they are especially abundant in the nervous system: gangliosides constitute $10-12 \%$ of total lipid content in neuronal membrane [4]. Glycosphingolipids are involved in a variety of functions due to their structural heterogeneity and location in cellular membranes. These are broadly classified into two categories: structural and receptor. In their structural role, they modulate the structure and dynamics of the membrane in which they are embedded, and in their receptor role, they bind to a variety of exogenous and endogenous molecules [5-8]. In both cases, their action triggers a series of physiological and, in some cases, pathological, effects. Glycosphingolipids have been shown to aggregate with sphingomyelins and cholesterol in model fluid membranes and also to form lipid rafts in biological membranes [9-12]. In addition, they form domains known as glycosynapse, and these are independent of cholesterol [9]. The presence of gangliosides at the plasma membrane makes them a target for a variety of bacterial toxins for initial recognition and infection of the host cell [13].

\section{Importance of Atomic Level Structural Data for Understanding of Biological Processes}

Considering the importance of glycolipids at lipid bilayers, there have been significant efforts to understand their structure and dynamics in lipid bilayers. The structural characterization of glycosphingolipids at the atomic level is challenging because of the (1) conformational variability generated due to sugar-sugar and sugar-lipid glycosidic linkages, (2) the dependence of glycolipid presentation to binding partners upon the lipid environment in which they are embedded, and (3) their self aggregation/phase separation in fluid membranes $[14,15]$. Biophysical methods such as nuclear magnetic resonance spectroscopy, X-ray diffraction, electron paramagnetic resonance spectroscopy, and fluorescence spectroscopy have been used to understand the behavior of glycolipids at lipid bilayers. However, these 
TABLE 1: Summary of literature reporting simulations of glycolipid containing lipid bilayer.

\begin{tabular}{|c|c|c|}
\hline System studied & Summary of simulations & Reference \\
\hline \multicolumn{3}{|c|}{ MD simulations of glycolipids focusing on behavior of glycolipids at lipid bilayers } \\
\hline $\begin{array}{l}\text { Single GD1a in DMPC } \\
\text { bilayer }\end{array}$ & $\begin{array}{l}\text { One simulation of single GD1a in a bilayer composed of } 15 \text { DMPC molecules was } \\
\text { performed for } 0.6 \mathrm{~ns}\end{array}$ & {$[16]$} \\
\hline $\begin{array}{l}\text { Single GM1 in DPC } \\
\text { micelle }\end{array}$ & $\begin{array}{l}\text { Single GM1 and GM1-Os was simulated for } 1 \mathrm{~ns} \text { in } 84 \text { DPC micelle and water. The effect of } \\
\text { chain length of GM1 on the conformation and dynamics of head-group was also evaluated }\end{array}$ & {$[17]$} \\
\hline $\begin{array}{l}\text { Single GM1 in DMPC } \\
\text { bilayer }\end{array}$ & $\begin{array}{l}\text { One simulation of single GM1 in a bilayer composed of } 15 \text { DMPC molecules was performed } \\
\text { for } 1 \mathrm{~ns}\end{array}$ & {$[18]$} \\
\hline $\begin{array}{l}\text { Single GM1* in DOPC } \\
\text { bilayer }\end{array}$ & $3.8 \mathrm{~ns}$ simulations of $\mathrm{GM} 1^{*}$ in a system composed of 278 DOPC lipid bilayer & {$[19]$} \\
\hline $\begin{array}{l}\text { Single GM1 in DPPC } \\
\text { bilayer }\end{array}$ & $\begin{array}{l}11 \text { simulations of a GM1 in a bilayer composed of } 97 \text { DPPC were performed. The dynamics } \\
\text { of GM1 in lipid bilayer was compared with that of GM1-Os in water }\end{array}$ & {$[20]$} \\
\hline $\begin{array}{l}\text { Single GM3 in DMPC } \\
\text { bilayer }\end{array}$ & 30 ns long simulations of GM3 in DMPC bilayer and GM3-Os in water were performed & {$[14]$} \\
\hline $\begin{array}{l}\text { One } \mathrm{GM} 1 * \text { in each } \\
\text { monolayer of DOPC } \\
\text { molecules }\end{array}$ & $\begin{array}{l}40 \mathrm{~ns} \text { long simulations of a system composed of two GM1* molecules, one in each leaflet, in } \\
278 \text { DOPC lipid bilayer }\end{array}$ & {$[21]$} \\
\hline \multicolumn{3}{|c|}{ MD simulations of glycolipids focusing on study of glycolipid enriched microdomains } \\
\hline Pure GM3 bilayer & $\begin{array}{l}50 \mathrm{~ns} \text { simulation of a bilayer composed of } 128 \mathrm{GM} 3 \text { was performed as a model system to } \\
\text { study gangliosides aggregates }\end{array}$ & {$[22,23]$} \\
\hline Glucosyl-glycerol bilayer & $\begin{array}{l}\text { Five different sets of parameters were used to simulate bilayer composed of } 128 \\
\text { palmitoyl-glucosyl glycerols. Simulations were performed for } 10-25 \mathrm{~ns} \text {. The goal of study } \\
\text { was to find correct parameters set that reproduce experimental data }\end{array}$ & {$[24]$} \\
\hline $\begin{array}{l}\text { GalCer and DPPG } \\
\text { bilayer }\end{array}$ & $\begin{array}{l}\text { Lipid bilayers composed of } 1024 \text { molecules of DPPG and consisting of } 10 \% \text { and } 25 \% \\
\text { GalCer were simulated for } 10 \mathrm{~ns}\end{array}$ & {$[25]$} \\
\hline $\begin{array}{l}\text { Glucosyl and galactosyl } \\
\text { glycerolipid }\end{array}$ & $\begin{array}{l}\text { Bilayers composed of } 128 \text { glucosyl and galactosyl glycerolipids were simulated. Structural } \\
\text { and dynamical properties of these bilayers were compared with that of PC and PE bilayers }\end{array}$ & {$[26]$} \\
\hline $\begin{array}{l}4 \text { GM1 in a bilayer } \\
\text { composed of POPC and } \\
\text { cholesterol }\end{array}$ & $\begin{array}{l}40 \text { ns simulation of ternary bilayer composed of GM1, cholesterol and POPC was } \\
\text { performed. The structure and dynamics of this lipid bilayer was compared with that of pure } \\
\text { and binary lipid bilayers }\end{array}$ & {$[27]$} \\
\hline $\begin{array}{l}\text { Varying concentrations } \\
\text { of GM1 in DPPC bilayer }\end{array}$ & $\begin{array}{l}\text { Lipid bilayers composed of varying concentrations of GM1 in DPPC bilayer }(\sim 5-25 \%) \text { were } \\
\text { studied using } 20 \text { ns MD simulations. Simulations were performed for systems containing } \\
\text { GM1 in single as well as both leaflets of bilayer }\end{array}$ & {$[28]$} \\
\hline $\begin{array}{l}\text { Simulations of GalCer in } \\
\text { a raft like membrane }\end{array}$ & $\begin{array}{l}200 \text { ns simulations of GalCer in raft like membrane composed of POPC, PSM, and } \\
\text { cholesterol }\end{array}$ & {$[29]$} \\
\hline
\end{tabular}

methods do not provide atomic level insights into the conformation and dynamics of a glycolipid at lipid bilayer and its dependence on the bilayer it is anchored in. Computer simulations, on the other hand, provide atomic level insights taking clues from the biophysical techniques on the structure and dynamical properties of glycolipid anchored on the lipid bilayer.

\section{Overview}

This paper focuses on the important insights gained from MD simulations into the structure, dynamics, and modulation of glycolipids at model lipid bilayers. There are fewer simulation studies on model membranes containing glycolipids (Table 1) when compared to those on phospholipids, sphingolipids, and cholesterol-containing lipid bilayers with or without protein [30-34]. This is not surprising considering the complexity of carbohydrate structures, the consequent inadequacy of their representation in the residue libraries of most of the simulation packages, and the unavailability of standard force fields. Most of the glycolipid simulations have considered the systems containing the gangliosides GM1, GD1a, and GM3. These have been extensively characterized by experimental techniques [9-11] due to their associations in several pathological processes [13]. Simulations whose focus was to understand the structure and dynamics of ganglioside(s) in lipid bilayer are reviewed first. The outcome of such simulations will help in understanding the presentation of gangliosides to their interacting 


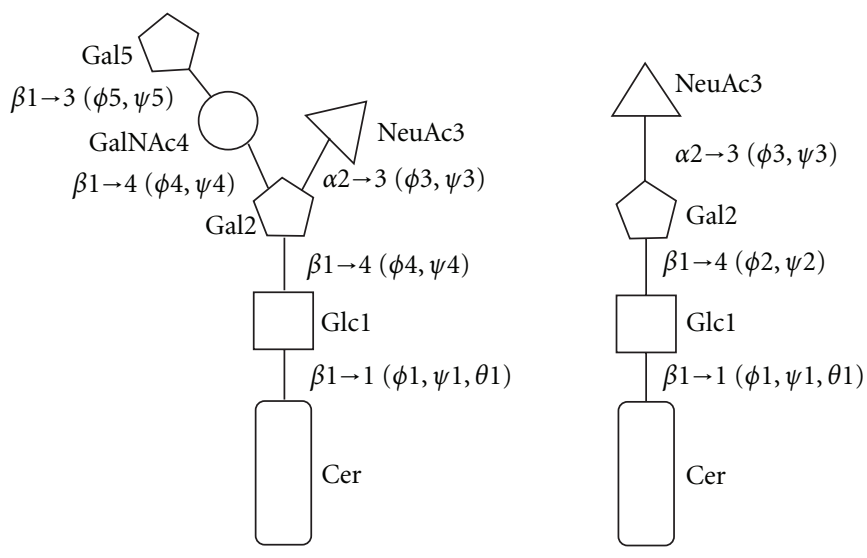

FIGURE 1: Schematic representation of the structure and dihedral nomenclature of glycosphingolipid molecules described in this paper. For sugar-sugar linkages other than those of NeuAc3, $\phi 1=\mathrm{H} 1-\mathrm{C} 1-\mathrm{O}-\mathrm{CX}^{\prime}$ and $\psi 1=\mathrm{C} 1-\mathrm{O}-\mathrm{CX}^{\prime}-\mathrm{HX}^{\prime}$; for those of NeuAC3, $\phi 1=\mathrm{C} 1-\mathrm{C} 2-\mathrm{O}-\mathrm{C} 3{ }^{\prime}$ and $\psi 1=\mathrm{C} 1-\mathrm{O}-\mathrm{CX}^{\prime}-\mathrm{HX}^{\prime}$. For sugar-ceramide linkages, $\phi 1=\mathrm{Glc1}: \mathrm{H} 1-\mathrm{Glc1}$ :C1-Glc1:O1-Cer:C1, $\psi 1$ = Glc1:C1-Glc1:O1-Cer:C1-Cer:C2, and $\theta 1=$ Glc1:O1-Cer:C1-Cer:C2-Cer-C3.

macromolecular partners. This is followed by a review of the simulations of bilayers with higher concentrations of glycolipids, where the primary focus was to understand the structure and dynamics of glycolipid-enriched micro domains. The paper is concluded by summary and future perspectives.

\section{Single Glycolipid in Lipid Bilayer}

Although initial efforts were limited to small systems $(<20$ lipids) and relatively shorter time scales (subnanosecond), considerably larger systems ( 200 lipids $)$ have now been simulated and for longer time scales (40 ns). An increase in the available computational power has also enabled the study of dynamics of the same systems with multiple starting conformations of glycolipid head groups.

4.1. Orientation and Exposure of Head-Group. As the glycolipid receptor function is governed by the orientation and exposure of head-group from lipid bilayer, it is important to understand the extent and orientation with which glycolipids can be presented "out" of a bilayer. This depends upon the nature of lipid moiety of glycolipids and of the bilayer in which they are present [36]. If the carbohydrate headgroup is "close" to the lipid surface, it is not possible for the macromolecule to access, bind, and initiate downstream physiological/pathological events. MD simulations of GM1 and of GM3 in a variety of membranes have provided valuable insights into their exposure at lipid bilayer.

It was first shown from MD simulations of GM1 in a DMPC bilayer that NeuAc3 (nomenclature as in Figure 1) extends in to the aqueous phase [18]. In agreement with this observation, multiple simulations of GM1 in a DPPC bilayer starting from different conformations showed that the phosphorous-NeuAc3 peak-to-peak distance, as calculated from density profile, is in the range of 0.21 to $0.53 \mathrm{~nm}$, indicating that NeuAc3 is extended beyond the phosphorous atoms of DPPC [20]. This was also supported by the observation that the orientation of Gal2-NeuAc3 branch of GM1 remains more or less parallel to bilayer normal (inferred based on the angle subtended by the vector Gal2:C1 $\rightarrow$ NeuAc3:C5 which is in the range of $20-56^{\circ}$; see Figure 2). However, in a recent simulation of GM1* in a DOPC bilayer, it has been shown that NeuAc3 lies always inside the headgroup region of the membrane, with Gal2: NeuAc3 oriented at $\sim 45^{\circ}$ with respect to bilayer normal [21].

GM1 is a pentasaccharide (Figure 1), branched from Gal2 residue, one branch being the single NeuAc3, while the other, the disaccharide GalNAc4-Gal5. The Gal2-GalNAc4Gal5 branch has been observed to be on the surface of the bilayer [18]. However, a recent study indicated two kinds of extension of this branch (Gal2-GalNAc4-Glc5) out of the bilayer; these were considered as different "equilibrium arrangements" [21]. The Gal2-GalNAc4-Glc5 branch of a GM1* molecule is extended beyond the bilayer surface, whereas that of other $\mathrm{GM}^{*}$ is on the surface. In simulations wherein GM1 is present in only one leaflet of the DPPC bilayer, Gal2-GalNAc4-Gal5 branch was found to be extended well beyond the surface of bilayer in two out of eight simulations, while it remains on the surface in rest of the simulations, as evident from phosphorousGalNAc4/Gal5 distances [20]. This branch (Gal2-GalNAc4Gal5) was observed to be oriented at an angle of 54-101 ${ }^{\circ}$ with respect to bilayer normal (i.e., parallel with respect to bilayer surface) (based on the angle subtended by the Gal2 : C1 $\rightarrow$ Gal5 : C4 vector; see Figure 2) [20]. Simulation of GM1 in a DPC micelle showed a membrane surface orientation in spite of using a starting configuration in which GM1 is extended well beyond the micellar surface [17]. MD simulations of GM3 in a DMPC bilayer showed that NeuAc3 is fully extended out of bilayer. Although not quantified for entire trajectory, one of the snapshots of the trajectory showed a parallel orientation of GM3 head-group with respect to bilayer normal [14].

4.2. Influence of the Membrane Environment on the Conformation of Head-Group. The conformational dynamics of glycolipids in a membrane is compared with its dynamics in water 


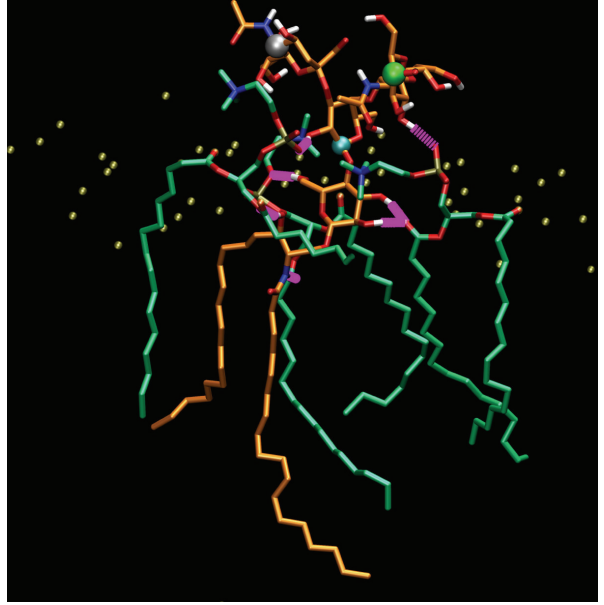

(a)

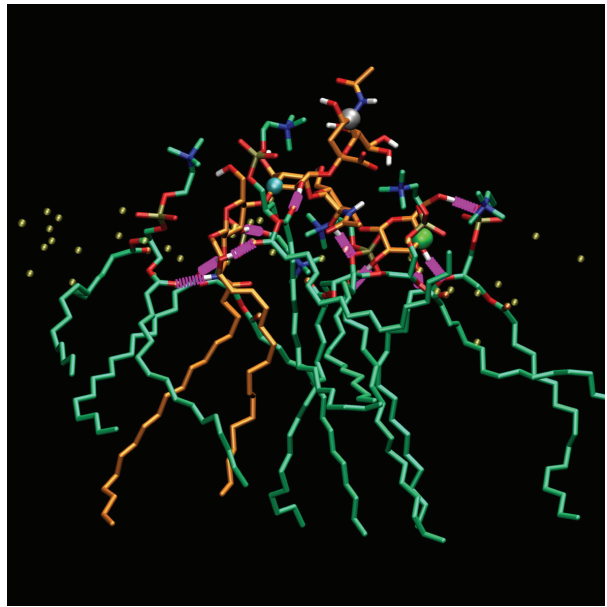

(b)

FIGURE 2: Two representative orientation/organization of GM1 in a DPPC bilayer. The angle formed by the vector Gal2: C1 $\rightarrow$ Gal5: C4 with the $Z$-axis is $56^{\circ}$ (a) and $105^{\circ}$ (b). Likewise, the angle formed by the vector Gal2: C1 $\rightarrow$ NeuAc3: C5 with the Z-axis is $39^{\circ}$ (a) and $47^{\circ}$ (b). GM1 forms 7 (a) and 10 (b) hydrogen bonds with DPPC. The carbonyl oxygen atoms of DPPC fatty acid chains are rendered as tiny yellow spheres and these represent the approximate lipid-solvent interface. Carbon atoms of GM1 and DPPC molecules are rendered in green and orange sticks. The atoms Gal2: C1, Gal5: C4, and NeuAc3 : C5 are rendered as cyan, green, and gray spheres, respectively. Hydrogen bonds between GM1 and DPPC molecules are rendered with thick magenta connector. The snapshots, rendered using VMD [35], are from the simulations with starting conformation C (at $4 \mathrm{~ns}$; (a)) and G (at $7 \mathrm{~ns}$; (b)) [20].

to understand how the membrane environment influences the conformation of the glycan head-group $[14,17,20]$. Simulations have shown that overall, the conformational dynamics of GM1 in lipid environment is somewhat lower than that in water $[17,20]$. Clearly, anchoring the glycolipid in a bilayer imposes restriction on the dynamics of glycosidic dihedral angles. On the time scale of the simulations, certain conformations (as represented by $\phi$ and $\psi$ ) that are accessed when the glycolipid oligosaccharide is in solution are not accessed when the glycolipid is anchored in a bilayer. MD simulations of GM3 showed a restricted accessibility and did not access region corresponding to $(\phi 2, \psi 2)=(180,0)$ that was accessed by GM3-Os in water for a similar time scale [14]. Simulations of GM1 in a DPPC bilayer and of GM1-Os in water showed that the linkages $(\phi 2, \psi 2)$ and $(\phi 5, \psi 5)$ display reduced flexibility when GM1 is anchored in the bilayer [20]. Similarly, simulations of GM1 with varying lengths of ceramide showed restricted flexibility of one or more pairs of dihedrals $(\phi 2, \psi 2),(\phi 3, \psi 3)$, and $(\phi 4, \psi 4)$ when GM1 is anchored in a DPC micelle as compared to GM1-Os in solution [17]. Thus, anchoring of GM1 in a membrane environment reduces the overall degrees of freedom available to the carbohydrate head-group [20].

The conformation of the ceramide-saccharide linkage, represented by the dihedral angles $(\phi 1, \psi 1$, and $\theta 1)$, has also been investigated using MD simulations of GM1 in a DPPC lipid bilayer [20] or a DPC micelle [17]. Both of these studies showed that $\phi 1$ is somewhat restricted, while $\psi 1$ and $\theta 1$ access several conformations. MD simulations of GM1 in a DPC micelle showed that the conformational dynamics of $\psi 1$ and $\theta 1$ is dependent on the hydrophobic chain length of the ceramide moiety. Changes in the dihedral angles $(\phi 1, \psi 1$, and $\theta 1)$ were naively expected to change the orientation of the head-group, but simulations of GM1 in a DPPC bilayer showed that it is not so; in fact, to a certain extent, it influenced the orientation of the ceramide chain.

4.3. Solvent-Exposed Surface Area. The solvent-exposed surface area of a glycolipid in a liquid crystalline bilayer is a property that determines the exposure of the glycolipid to water and, in turn, to the macromolecule. The solventexposed surface of a glycolipid when anchored on lipid bilayer can be compared to that of the corresponding glycolipid head-group in solution to gain insights into the extent to which exposure is masked in the presence of bilayer. Multiple simulations of GM1 in a DPPC bilayer showed that the solvent exposed surface area of NeuAc3 of GM1 in bilayer is comparable to that of the GM1Os in water [20]. This indicates that the presence of a lipid bilayer is not significantly altering the "accessibility" of NeuAc3 to the macromolecule (Figures 3(c), 3(d), and $3(\mathrm{e})$ ). However, the solvent-exposed surface areas of Glc1 and Gal2 are significantly reduced by the presence of the DPPC bilayer, while large deviations in the SASA of Gal5 and GalNAc4 are observed for different simulated systems, indicating that the extent to which the bilayer masks these residues is quite variable (Figures 3(c), 3(d), and 3(e)) [20]. MD simulations of GM1 along with NMR paramagnetic relaxation enhancement experiment showed that the presence of bilayer reduces the accessible surface area by $75 \%, 66 \%, 16 \%, 19 \%$, and $10 \%$ for Glc1, Gal2, NeuAc3, GalNAc4, and Gal5 residues, respectively [15]. Results of MD simulations from the same group showed that the presence of 


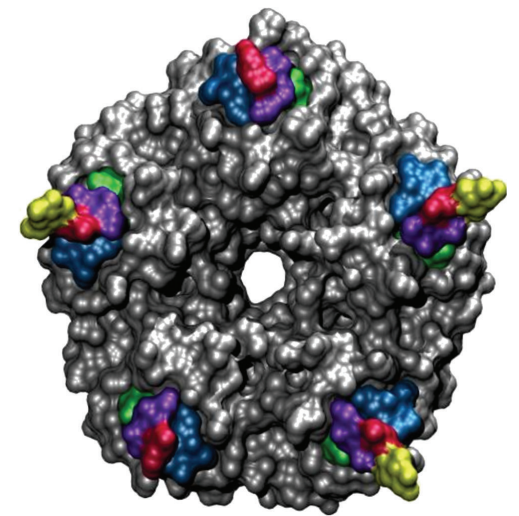

(a)

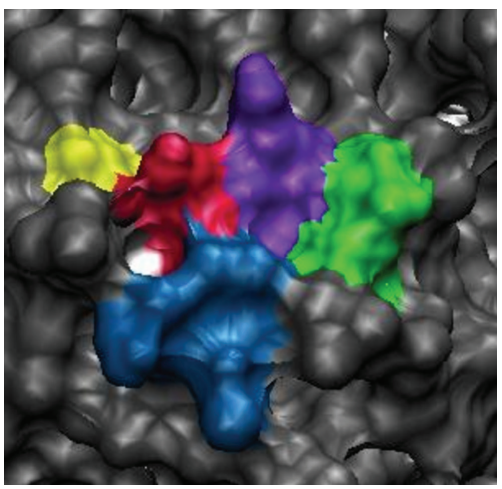

(c)

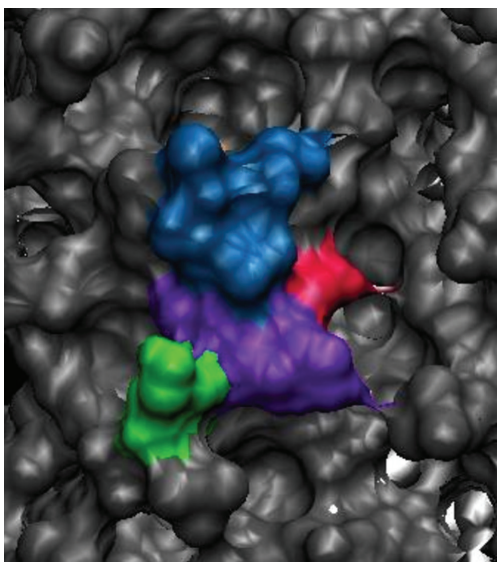

(e)

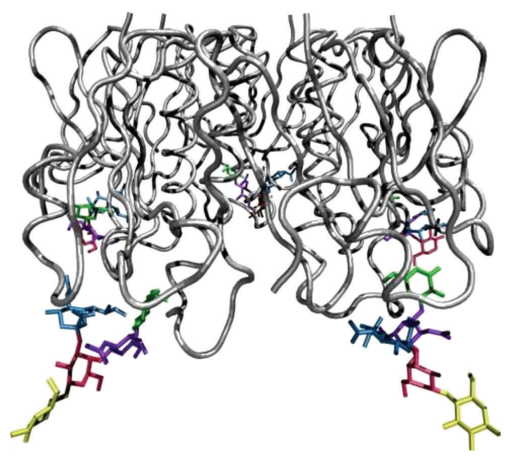

(b)

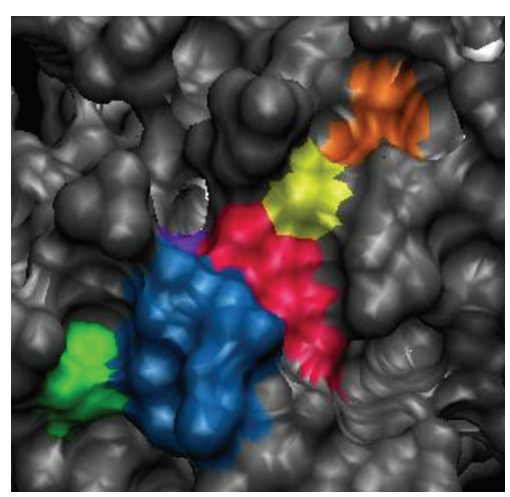

(d)

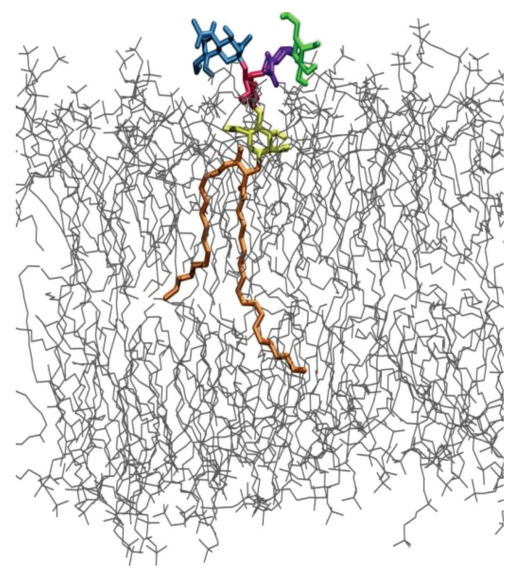

(f)

FIGURE 3: Surface (a) and ribbon + stick (b) rendering of the cholera toxin-GM1 complex, viewed either along (a) or perpendicular (b) to the molecular 5-fold axis (PDB id 3CHB). Surface renderings (panels (c), (d), and (e)) and stick diagram (panel (f)) of GM1 embedded in a DPPC bilayer taken from MD simulations starting with conformation B (panel (c); at $4 \mathrm{~ns}$ ), G (panel (d); at $10 \mathrm{~ns}$ ), or C (panels (e) and (f); at $4 \mathrm{~ns}$ ) [20]. In the snapshots shown in panels (c), (d), and (e)/(f), the SASA of NeuAc3 is comparable to each other and is within the range 182-200 $\AA^{2}$. However, there is a marked difference in the exposure of Gal5, and its SASA varies widely: $101 \AA^{2}$ (panel (c)), $14 \AA^{2}$ (panel (d)) and $151 \AA^{2}$ (panels (e)/(f)). Color code: Glc1, yellow; Gal2, red; NeuAc3, cyan; GalNAc4, magenta; Gal5, green; Ceramide, orange; cholera toxin/DPPC, grey. The molecules were rendered using VMD [35].

GM3 in a DMPC bilayer reduces the accessible surface area by $73 \%, 27 \%$, and $5 \%$ for Glc1, Gal2, and NeuAc3 residues [14]. Overall, these observations indicate that the presence of lipid bilayer reduces the accessible surface area of one or two sugar molecules that are linked to lipid, while the other, especially NeuAc3, is solvent exposed.
The crystal structures of a few protein molecules bound to either GM1-Os or GM3-Os have been determined. Two monosaccharide moieties of GM1, Glc1, and Gal2, make no significant or direct contact with cholera toxin (PDB: 3CHB; see Figures 3(a) and 3(b) [37] or SV40 capsid protein VP1 (PDB: 3BWR) [38]. Of the other three monosaccharides, 
the terminal Gal5 and NeuAc3 form several polar as well as nonpolar interactions in both the VP1 and cholera toxin complexes. Similarly, a combined NMR spectroscopy and modeling study showed that galectin-1 forms favorable interactions with Gal5, GalNAc4 and NeuAc3 compared to Gal2 and Glc1 [39]. Thus, the accessibility of NeuAc3, GalNAc4, and Gal5 seems to be essential for cholera toxin and VP1 to bind to GM1 [37, 38]. From MD simulations of GM1 reported by various laboratories, it is evident that Glc1 and Gal2 are not exposed to bilayer surface for macromolecules to bind (Figures 3(c), 3(d), and 3(e)). However, there are different views about the exposure of NeuAc3 and Gal5. It might be expected that the availability of either the terminal saccharide at the bilayer may provide initial interactions with macromolecules, followed by protein-assisted changes in the orientation of GM1 to provide the full array of interactions. The crystal structures of GM3 with sialoadhesin (PDB: 1QFO) [40] and enterotoxin (PDB: 1SE3) [41] also showed that most of the interactions with the protein are provided by external NeuAc3, while Glc1 and Gal2 provide no or few interactions.

4.4. Modulation of Lipid Membrane. The hydrophobic tail and the polar head-group of a glycolipid are different from those of the lipids that constitute the bilayer in which it is embedded. Thus, the glycolipid is expected to affect the structure and dynamics of neighboring lipid molecules. MD simulations of GD1a in a DMPC bilayer showed that the mean squared displacement of choline nitrogen atoms and the terminal carbon atom of the hydrophobic tail of DMPC molecules in the neighborhood of GD1a is higher compared to that of the nonneighboring DMPC and of DMPC molecules of a monolayer without GD1a [16]. A similar effect was observed in the MD simulations of GM1 in DMPC bilayer [18]. In multiple simulations of GM1 in DPPC bilayer, it was observed that when the properties of the entire bilayer are compared with or without GM1, the bilayer thickness is similar to DPPC bilayer without GM1 [20]. The orientation of choline P-N vector and the order parameter of DPPC in a monolayer without GM1 are similar to those in a monolayer with GM1, indicating the absence of any effect on the bulk properties of bilayer. However, when the properties of DPPC molecules in the neighborhood of GM1 are compared with those of the same number of nonneighboring DPPC molecules, the order parameters of GM1neighborhood DPPC are found to be significantly lower than those of the nonneighbor DPPC molecules. Similarly, the orientations of the P-N vector of DPPC molecules in the vicinity of GM1 are also significantly perturbed compared to those of DPPC which are not in the vicinity. These two observations show that the GM1 molecule induces significant disorder in the dynamics of neighboring lipid molecules. Similar observations were made from the MD simulations of the ganglioside GM1* in DOPC bilayer [21].

The differences in hydration and the number of intraand intermolecular hydrogen bonds formed by GM1-Os in water and GM1 in lipid bilayer have also been investigated [20]. The number of water molecules (within $0.35 \mathrm{~nm}$ of glycan oxygen atoms) hydrating GM1 in DPPC bilayer was found to be nearly half of those hydrating GM1-Os in water. Concomitantly, the number of hydrogen bonds formed with water also reduced when GM1 is anchored on DPPC bilayer, part of which are replaced by hydrogen bonds with DPPC.

\section{Multiple Glycolipids in Lipid Bilayer}

Glycosphingolipids have been shown to aggregate in model membranes containing sphingomyelins and cholesterol and are present in lipid rafts in biological membrane. This has led to MD simulations studies of complex (i.e., containing multiple lipid types) systems which mimic lipid rafts [27, 29]. Simulations of a binary lipid mixture where glycolipids are mixed with one other kind of lipid have also been reported; the focus is more on modulation of the lipid bilayer induced by the presence of glycolipids $[25,28]$. In addition to this, MD simulations of pure glycolipid bilayer have also been performed to investigate the properties of pure model membranes $[22,24,26]$ and to investigate the validity of parameter set [24]. In this part of the paper, insights gained from MD simulations of mixed lipid bilayers are reviewed focusing on the modulation of pure/mixed lipid bilayers induced by the presence of more than one glycosphingolipid.

5.1. Effect on Bilayer Properties. MD simulations of varying concentrations of GM1 and DPPC in symmetric (i.e., GM1 is present in both the leaflets) bilayers showed that the presence of $5 \%$ to $25 \%$ GM1 in DPPC bilayer decreases surface area per lipid and increases deuterium order parameter and peak-to-peak distance of DPPC (Figure 4). This effect is less pronounced in an asymmetric (i.e., GM1 is present in only one of the leaflets) lipid bilayer [28]. These MD simulations also showed that the presence of GM1 broadens the lipid water interface, due to the larger sugar headgroup. Incorporation of 4 GM1 molecules in one leaflet of a bilayer composed of POPC and cholesterol (32 and 12 molecules, respectively; area: $53 \pm 2 \AA^{2}$ ) did not significantly alter either the surface area (area per lipid of POPC + cholesterol is $54 \pm 2 \AA^{2}$ ) or the bilayer thickness [27]. It can be anticipated that the incorporation of cholesterol might have already ordered the system sufficiently. Incorporation of $5 \%$ GalCer in a membrane composed of POPC, cholesterol, and sphingomyelin increases the bilayer thickness although the surface area of the lipid molecules is not reduced [29].

5.2. Clustering. There are at least two studies where MD simulations of glycosphingolipids have been performed in multicomponent model membranes to gain insights into the interactions and organization of glycosphingolipids with raft lipids $[27,29]$. From the radial distribution function of various lipid molecules in a four component bilayer, it has been shown that cholesterol tends to organize themselves forming a local structure and that GalCer and PSM favor cholesterol as compared to POPC [29]. A somewhat similar behavior was also observed in the simulation of a ternary lipid bilayer containing POPC, cholesterol, and GM1 [27]. Cholesterol was observed to form local clusters in the leaflet of bilayer 


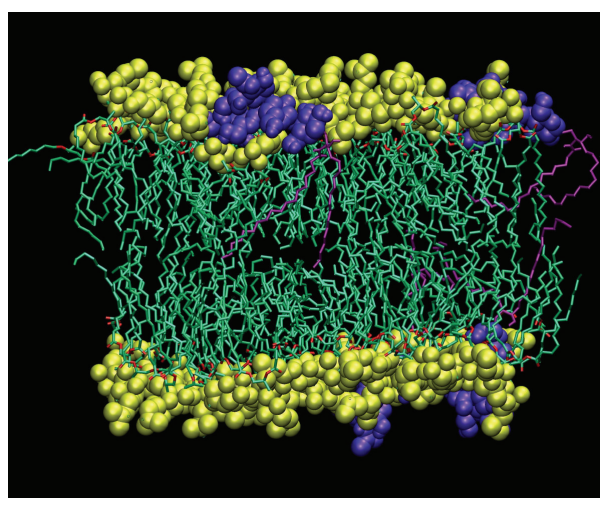

(a)

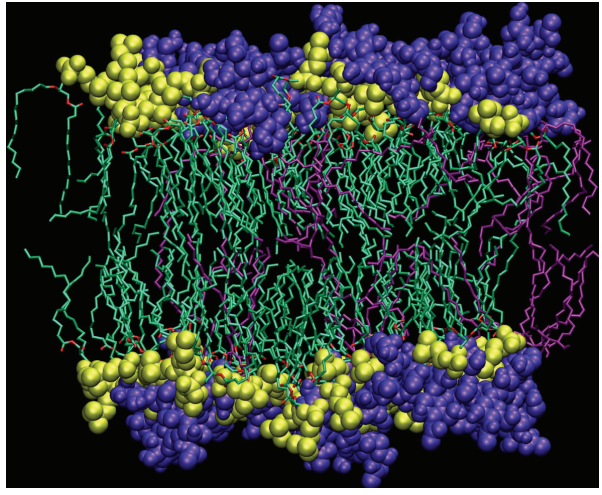

(b)

FIGURE 4: Snapshots of binary lipid bilayers composed of DPPC and GM1 in the ratio $94: 4$ (a) or $78: 20$ (b). A decrease in the DPPC: GM1 ratio leads to a broadening of the polar interface and thickening of the bilayer. The snapshots are from the simulations sym $94: 4$ (at $14 \mathrm{ns)}$ and sym78:20 (also at $14 \mathrm{ns)} \mathrm{[28]} \mathrm{and} \mathrm{are} \mathrm{rendered} \mathrm{using} \mathrm{VMD} \mathrm{[35].} \mathrm{Polar} \mathrm{atoms} \mathrm{of} \mathrm{DPPC} \mathrm{and} \mathrm{head-groups} \mathrm{of} \mathrm{GM1} \mathrm{are} \mathrm{rendered} \mathrm{as} \mathrm{yellow}$ and blue spheres, respectively. Other carbon atoms of DPPC and GM1 are rendered as green and magenta sticks, respectively.

containing GM1 compared to the leaflet which did not. Disruption of microdomain integrity by 2-hydroxypropyl$\beta$-cyclodextrin or filipin III has been shown to reduce the binding affinity of galectins 1 and 3 to cholesterol-depleted neuroblastoma cells. This observation shows the importance of modulation of glycolipid presentation by composition of the lipid matrix, especially in the presence or absence of cholesterol, in which it is embedded [42]. The number of atomic contacts between GM1 and cholesterol was observed to be higher than those of GM1 and POPC in spite of the higher number of POPC compared to cholesterol in the model membrane. There were no hydrogen bonds between GM1 and cholesterol although a few water-mediated hydrogen bonds are noticed, indicating that the preferential mode of interaction between GM1 and cholesterol is hydrophobic. MD simulations of four-component lipid bilayer showed that the number of cholesterol-POPC hydrogen bonds are more than those of either cholesterol-GalCer or cholesterol-PSM [29].

5.3. Organization. As MD simulations of glycosphingolipids at lipid bilayer provided evidence of formation of local structures, the nature of interaction between glycolipids and raft lipids (sphingolipids and/or cholesterol) were visually investigated [27, 29]. From the MD simulations of GalCer in three-component model membrane, it was observed that GalCer, despite having the same fatty acid chain as POPC, interdigitates into the opposing leaflet of the bilayerreducing lateral diffusion. It was also observed that the glycan head-group of GalCer remains at a fixed position with respect to cholesterol and POPC, slightly below the POPC head-group but above cholesterol. This arrangement hides the hydrophobic part of cholesterol from water, providing a shielding effect [29]. However, this cholesterol-GalCer interaction might lock GalCer in a conformation that is not accessible to macromolecular binding partners as proposed from the observation that a major fraction of various glycolipids in glycosphingolipid/cholesterol model membrane is not accessed by macromolecules [43]. MD simulations of GM1 in a model membrane composed of cholesterol and POPC showed that the tendency to form cholesterol-rich domain is observed only in the presence of GM1 [27]. POPC molecules were ordered between two GM1 molecules, and several cholesterol molecules were stacked around GM1. The sterol ring of cholesterol interacts with its face opposing the ceramide chain. It has also been suggested that the presence of GM1 may order the cholesterol molecules and provide a route to the formation of ordered domains.

\section{Summary and Future Perspectives}

Overall, molecular dynamics simulations have provided valuable and specific insights into the organization of glycolipids in single component and raft-like membranes, which is difficult to obtain from other biophysical techniques. Although the time scale and composition of simulations are far from being realistic, studies performed so far have broadened our understanding of the behavior of glycolipids at lipid bilayer.

Although there have been significant advances in the MD simulations of the structure and dynamics of model membranes, there are fewer reports on the simulations of glycolipids. Development of easily usable and largely applicable force-fields of carbohydrates with lipid parameters certainly enhances interest in performing simulations of model membrane composed of glycolipids. As there are fewer simulations reporting structure and dynamics of raftlike membrane, in the near future, there will be efforts to study the dynamics of proteins in raft-like membranes. Future efforts will also be directed towards an understand -ing of the interactions of glycolipids with their interacting partners at the lipid bilayer. The energetics of glycolipid insertion and compatibility in a variety of lipid bilayers certainly is an urgent need. MD simulations of very large 
systems will certainly help to understand the mesoscopic properties of glycolipid containing membrane.

\section{Abbreviations}

\begin{tabular}{|c|c|}
\hline DMPC: & Dimyristoylphosphatidylcholine \\
\hline DOPC: & Dioleoylphosphatidylcholine \\
\hline DPC: & Dodecylphosphocholine \\
\hline DPPC: & Dipalmitoylphosphatidylcholine \\
\hline Gal: & Galactose \\
\hline GalCer: & Galactosylceramide \\
\hline GalNAc: & $\mathrm{N}$-acetylgalactosamine \\
\hline GD1a: & $\begin{array}{l}\text { NeuAc6- } \alpha 2,3-\text { Gal5- } \beta 1,3-G a l N A c 4-\beta 1,4- \\
(\text { NeuAc3- } \alpha 2,3) \text {-Gal2- } \beta 1,4-G l c 1-\beta 1,1-\text { Cer }\end{array}$ \\
\hline Glc: & Glucose \\
\hline GM1: & $\begin{array}{l}\text { Gal5- } \beta 1,3-G a l N A c 4-\beta 1,4-(N e u A c 3-\alpha 2,3)- \\
\text { Gal2- } \beta 1,4-G l c 1-\beta 1,1-C e r\end{array}$ \\
\hline GM1*: & $\begin{array}{l}\text { Glc5- } \beta 1,3 \text {-GalNAc4- } \beta 1,4-(\text { NeuAc3- } \alpha 2,3)- \\
\text { Gal2- } \beta 1,4-G l c 1-\beta 1,1-\text {-Cer }\end{array}$ \\
\hline GM1-Os: & $\begin{array}{l}\text { Gal5- } \beta 1,3-G a l N A c 4-\beta 1,4-(N e u A c 3-\alpha 2,3)- \\
\text { Gal2- } \beta 1,4-G l c 1\end{array}$ \\
\hline GM3: & NeuAc3- $\alpha 2,3-G a l 2-\beta 1,4-G l c 1-\beta 1,1-C e r$ \\
\hline GM3-Os: & NeuAc3- $\alpha 2,3-G a l 2-\beta 1,4-G l c 1$ \\
\hline MD: & Molecular dynamics \\
\hline NeuAc: & $\mathrm{N}$-acetylneuraminic acid \\
\hline PC: & Phosphatidylcholine \\
\hline PE: & Phosphatidylethanolamine \\
\hline POPC: & Palmitoyloleoylphosphatidylcholine \\
\hline PSM: & Palmitoyl sphingomyelin \\
\hline SASA: & Solvent accessible surface area. \\
\hline
\end{tabular}

\section{References}

[1] A. Varki, Essentials of Glycobiology, Cold Spring Harbor Laboratory, New York, NY, USA, 2nd edition, 2008.

[2] V. S. R. Rao, Conformation of Carbohydrates, CRC Press, New York, NY, USA, 1998.

[3] R. W. Ledeen and G. Wu, "Nuclear sphingolipids: metabolism and signaling," Journal of Lipid Research, vol. 49, no. 6, pp. 1176-1186, 2008.

[4] E. Posse de Chaves and S. Sipione, "Sphingolipids and gan -gliosides of the nervous system in membrane function and dysfunction," FEBS Letters, vol. 584, no. 9, pp. 1748-1759, 2010.

[5] J. Kopitz, C. Von Reitzenstein, S. André et al., "Negative regulation of neuroblastoma cell growth by carbohydrate-dependent surface binding of galectin-1 and functional divergence from galectin-3," Journal of Biological Chemistry, vol. 276, no. 38, pp. 35917-35923, 2001.

[6] J. Kopitz, S. André, C. Von Reitzenstein et al., "Homodimeric galectin-7 (p53-induced gene 1) is a negative growth regulator for human neuroblastoma cells," Oncogene, vol. 22, no. 40, pp. 6277-6288, 2003.

[7] A. A. Vyas, H. V. Patel, S. E. Fromholt et al., "Gangliosides are functional nerve cell ligands for myelin-associated glycoprotein (MAG), an inhibitor of nerve regeneration," Proceedings of the National Academy of Sciences of the United States of America, vol. 99, no. 12, pp. 8412-8417, 2002.

[8] N. Ichikawa, K. Iwabuchi, H. Kurihara et al., "Binding of laminin-1 to monosialoganglioside GM1 in lipid rafts is crucial for neurite outgrowth," Journal of Cell Science, vol. 122, no. 2, pp. 289-299, 2009.
[9] B. Westerlund and J. P. Slotte, "How the molecular features of glycosphingolipids affect domain formation in fluid membranes," Biochimica et Biophysica Acta, vol. 1788, no. 1, pp. 194-201, 2009.

[10] G. Gupta and A. Surolia, "Glycosphingolipids in microdomain formation and their spatial organization," FEBS Letters, vol. 584, no. 9, pp. 1634-1641, 2010.

[11] A. Prinetti, N. Loberto, V. Chigorno, and S. Sonnino, "Glycosphingolipid behaviour in complex membranes," Biochimica et Biophysica Acta, vol. 1788, no. 1, pp. 184-193, 2009.

[12] L. Cantu, M. Corti, P. Brocca, and E. Del Favero, "Structural aspects of ganglioside-containing membranes," Biochimica et Biophysica Acta, vol. 1788, no. 1, pp. 202-208, 2009.

[13] T. D. Connell, "Cholera toxin, LT-I, LT-IIa and LT-IIb: the critical role of ganglioside binding in immunomodulation by Type I and Type II heat-labile enterotoxins," Expert Review of Vaccines, vol. 6, no. 5, pp. 821-834, 2007.

[14] M. I. Demarco and R. J. Woods, "Atomic-resolution conformational analysis of the $\mathrm{G}$ ganglioside in a lipid bilayer and its implications for ganglioside-protein recognition at membrane surfaces," Glycobiology, vol. 19, no. 4, pp. 344-355, 2009.

[15] M. L. DeMarco, R. J. Woods, J. H. Prestegard, and F. Tian, "Presentation of membrane-anchored glycosphingolipids determined from molecular dynamics simulations and NMR paramagnetic relaxation rate enhancement," Journal of the American Chemical Society, vol. 132, no. 4, pp. 13341338, 2010.

[16] D. Roy and C. Mukhopadhyay, "GDla in phospholipid bilayer: a molecular dynamics simulation," Journal of Biomolecular Structure and Dynamics, vol. 18, no. 4, pp. 639-646, 2001.

[17] S. V. Vasudevan and P. V. Balaji, "Dynamics of ganglioside headgroup in lipid environment: molecular dynamics simulations of GM1 embedded in dodecylphosphocholine micelle," Journal of Physical Chemistry B, vol. 105, no. 29, pp. 70337041, 2001.

[18] D. Roy and C. Mukhopadhyay, "Molecular dynamics simulation of GM1 in phospholipid bilayer," Journal of Biomolecular Structure and Dynamics, vol. 19, no. 6, pp. 1121-1132, 2002.

[19] M. Sega, P. Jedlovszky, and R. Vallauri, "Molecular dynamics simulation of GM1 gangliosides embedded in a phospholipid membrane," Journal of Molecular Liquids, vol. 129, no. 1-2, pp. 86-91, 2006.

[20] R. Y. Patel and P. V. Balaji, "Characterization of the conformational and orientational dynamics of ganglioside GM1 in a dipalmitoylphosphatidylcholine bilayer by molecular dynamics simulations," Biochimica et Biophysica Acta, vol. 1768, no. 6, pp. 1628-1640, 2007.

[21] P. Jedlovszky, M. Sega, and R. Vallauri, "GM1 ganglioside embedded in a hydrated DOPC membrane: a molecular dynamics simulation study," Journal of Physical Chemistry B, vol. 113, no. 14, pp. 4876-4886, 2009.

[22] M. Sega, R. Vallauri, P. Brocea, L. Cantù, and S. Melchionna, "Short-range structure of a GM3 ganglioside membrane: comparison between experimental WAXS and computer simulation results," Journal of Physical Chemistry B, vol. 111, no. 37, pp. 10965-10969, 2007.

[23] M. Sega, R. Vallauri, P. Brocca, and S. Melchionna, "Molecular dynamics simulation of a GM3 ganglioside bilayer," Journal of Physical Chemistry B, vol. 108, no. 52, pp. 20322-20330, 2004.

[24] T. Róg, I. Vattulainen, and M. Karttunen, "Modeling glycolipids: take one," Cellular and Molecular Biology Letters, vol. 10, no. 4, pp. 625-630, 2005. 
[25] T. Zaraiskaya and K. R. Jeffrey, "Molecular dynamics simulations andH NMR study of the GalCer/DPPG lipid bilayer," Biophysical Journal, vol. 88, no. 6, pp. 4017-4031, 2005.

[26] T. Róg, I. Vattulainen, A. Bunker, and M. Karttunen, "Glycolipid membranes through atomistic simulations: effect of glucose and galactose head groups on lipid bilayer properties," Journal of Physical Chemistry B, vol. 111, no. 34, pp. 1014610154, 2007.

[27] S. Mondal and C. Mukhopadhyay, "Molecular level investigation of organization in ternary lipid bilayer: a computational approach,” Langmuir, vol. 24, no. 18, pp. 10298-10305, 2008.

[28] R. Y. Patel and P. V. Balaji, "Characterization of symmetric and asymmetric lipid bilayers composed of varying concentrations of ganglioside GM1 and DPPC," Journal of Physical Chemistry B, vol. 112, no. 11, pp. 3346-3356, 2008.

[29] A. Hall, T. Róg, M. Karttunen, and I. Vattulainen, "Role of glycolipids in lipid rafts: a view through atomistic molecular dynamics simulations with galactosylceramide," Journal of Physical Chemistry B, vol. 114, no. 23, pp. 7797-7807, 2010.

[30] E. Lindahl and M. S. Sansom, "Membrane proteins: molecular dynamics simulations," Current Opinion in Structural Biology, vol. 18 , no. 4, pp. 425-431, 2008.

[31] P. S. Niemelä, M. T. Hyvönen, and I. Vattulainen, "Atom-scale molecular interactions in lipid raft mixtures," Biochimica et Biophysica Acta, vol. 1788, no. 1, pp. 122-135, 2009.

[32] F. Khalili-Araghi, J. Gumbart, P. C. Wen, M. Sotomayor, E. Tajkhorshid, and K. Schulten, "Molecular dynamics simulations of membrane channels and transporters," Current Opinion in Structural Biology, vol. 19, no. 2, pp. 128-137, 2009.

[33] M. L. Berkowitz, "Detailed molecular dynamics simulations of model biological membranes containing cholesterol," Biochimica et Biophysica Acta, vol. 1788, no. 1, pp. 86-96, 2009.

[34] S. A. Pandit and H. L. Scott, "Multiscale simulations of heterogeneous model membranes," Biochimica et Biophysica Acta, vol. 1788, no. 1, pp. 136-148, 2009.

[35] W. Humphrey, A. Dalke, and K. Schulten, "VMD: visual molecular dynamics," Journal of Molecular Graphics, vol. 14, no. 1, pp. 33-38, 1996.

[36] S. V. Evans and C. R. MacKenzie, "Characterization of proteinglycolipid recognition at the membrane bilayer," Journal of Molecular Recognition, vol. 12, no. 3, pp. 155-168, 1999.

[37] E. A. Merritt, P. Kuhn, S. Sarfaty, J. L. Erbe, R. K. Holmes, and W. G. J. Hol, "The $1.25 \AA$ resolution refinement of the cholera toxin B-pentamer: evidence of peptide backbone strain at the receptor-binding site," Journal of Molecular Biology, vol. 282, no. 5, pp. 1043-1059, 1998.

[38] U. Neu, K. Woellner, G. Gauglitz, and T. Stehle, "Structural basis of GM1 ganglioside recognition by simian virus 40," Proceedings of the National Academy of Sciences of the United States of America, vol. 105, no. 13, pp. 5219-5224, 2008.

[39] H. C. Siebert, S. André, S. Y. Lu et al., "Unique conformer selection of human growth-regulatory lectin galectin-1 for ganglioside GM1 versus bacterial toxins," Biochemistry, vol. 42, no. 50, pp. 14762-14773, 2003.

[40] A. P. May, R. C. Robinson, M. Vinson, P. R. Crocker, and E. Y. Jones, "Crystal structure of the N-terminal domain of sialoadhesin in complex with $3^{\prime}$ sialyllactose at $1.85 \AA$ resolution," Molecular Cell, vol. 1, no. 5, pp. 719-728, 1998.

[41] S. Swaminathan, W. Furey, J. Pletcher, and M. Sax, "Residues defining V $\beta$ specificity in staphylococcal enterotoxins," Nature Structural Biology, vol. 2, no. 8, pp. 680-686, 1995.

[42] J. Kopitz, M. Bergmann, and H.-J. Gabius, "How adhe -sion/growth-regulatory galectins- 1 and -3 attain cell specificity: case study defining their target on neuroblastoma cells (SK-N-MC) and marked affinity regulation by affecting microdomain organization of the membrane," IUBMB Life, vol. 62, no. 8, pp. 624-628, 2010.

[43] R. Mahfoud, A. Manis, B. Binnington, C. Ackerley, and C. A. Lingwood, "A major fraction of glycosphingolipids in model and cellular cholesterol-containing membranes is undetectable by their binding proteins," Journal of Biological Chemistry, vol. 285, no. 46, pp. 36049-36059, 2010. 


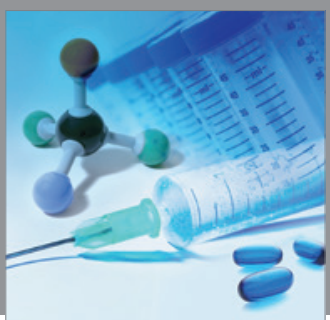

International Journal of

Medicinal Chemistry

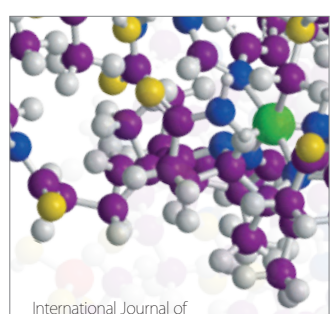

Carbohydrate Chemistry

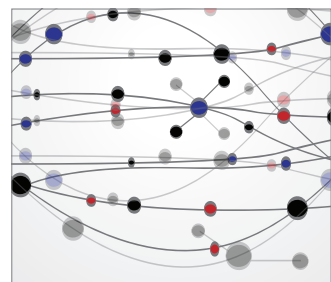

The Scientific World Journal
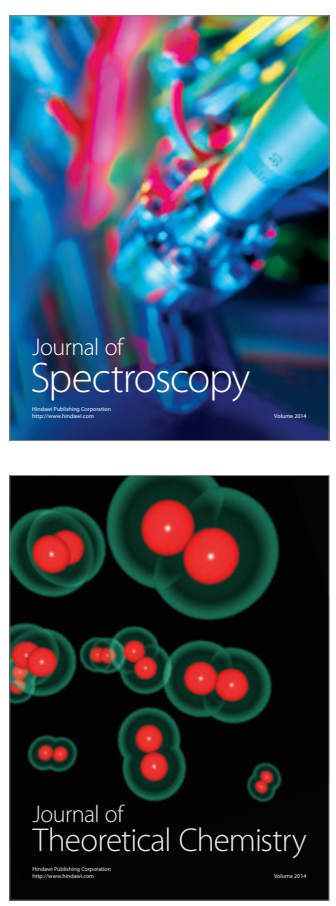
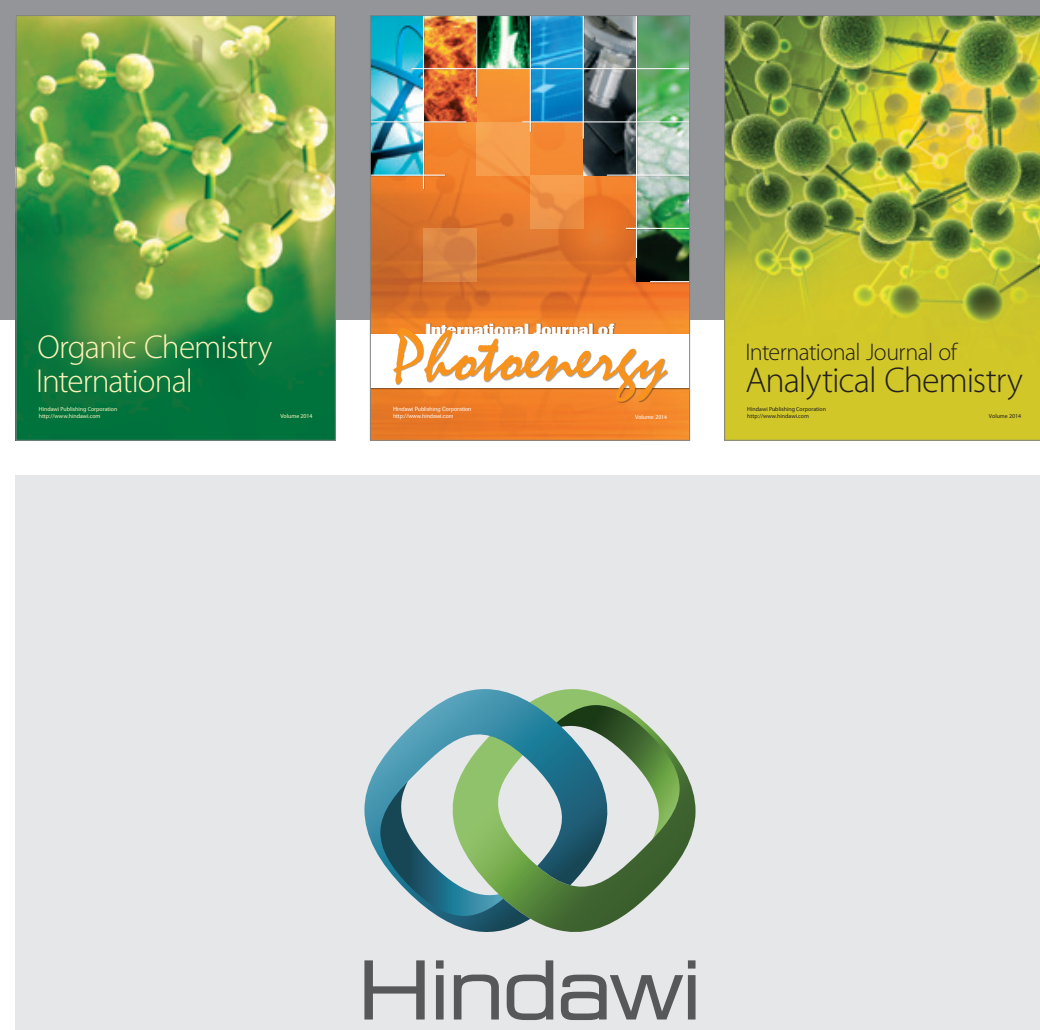

Submit your manuscripts at

http://www.hindawi.com
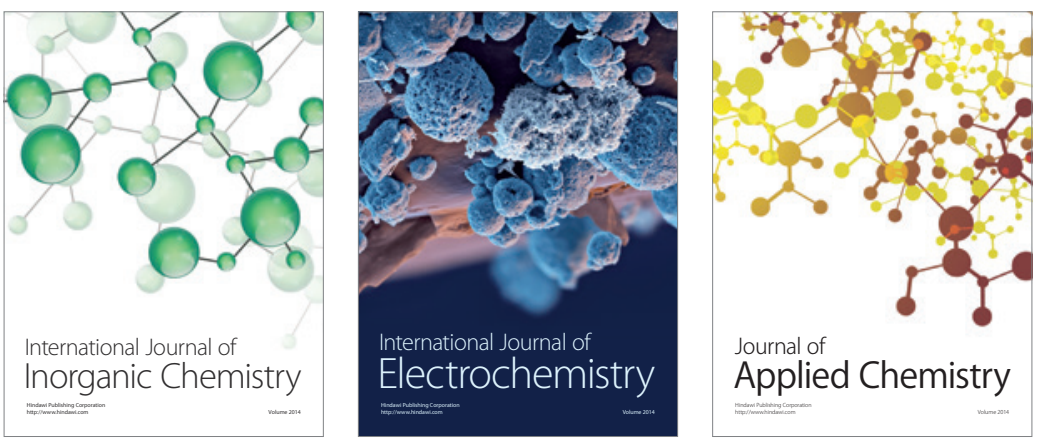

Journal of

Applied Chemistry
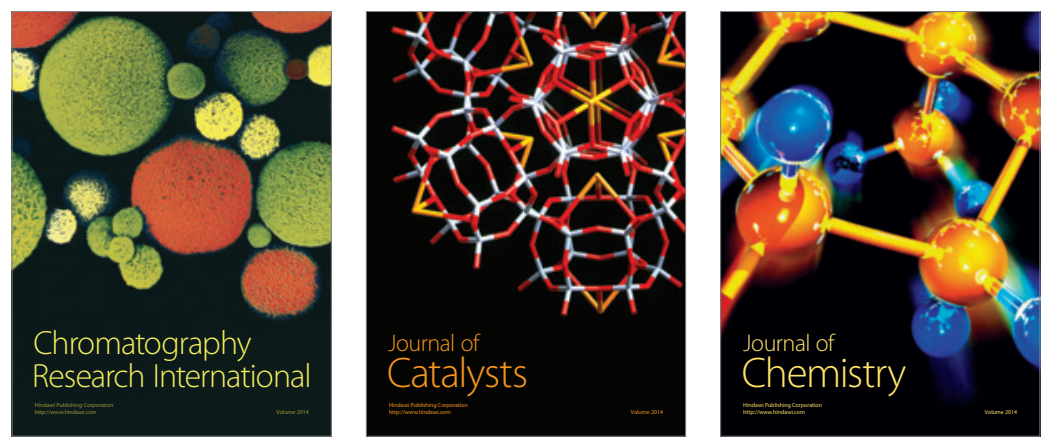
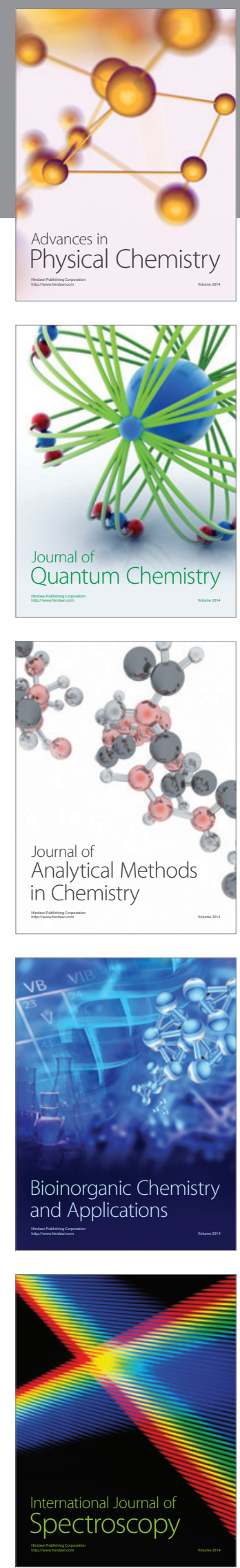